\title{
Field Experiences in Power Quality Problem Solving in Spain
}

\author{
Z. Larrabe ${ }^{1}$, J. M. López ${ }^{2}$ and G. Rojas ${ }^{3}$ \\ P.Q.C. Power Quality Control \\ Alameda de Mazarredo 43, 48009 Bilbao (Spain) \\ Phone: +34 944355 588, Fax: +34 944354 556, e-mail: ${ }^{1}$ zigor.larrabe@pqc.es, ${ }^{2}$ jonmikel.lopez@pqc.es, \\ garceran.rojas@pqc.es
}

\begin{abstract}
:
This paper is a summary of the experiences gathered by PQC during 10 years of field work in Power Quality problem solving in final customers. Topics which have been found to be in the origin of power quality problems will be analysed: earthing, UPS and emergency standby generators, harmonic distortion, transient overvoltages and monitoring.
\end{abstract}

Key Words: Power Quality, Low Voltage, Earthing

\section{Introduction}

Power Quality is a relatively new term that has arisen to refer to problems in the electricity supply. The goal of an electricity supply is to deliver a voltage waveform with proper magnitude and frequency, free from harmonic distortion or transient disturbances that would affect the loads.

Most of the papers about power quality deal with power quality issues in the utility side, mainly voltage sags. During the last ten years, P.Q.C. has been working in the opposite side for customers from different fields all around Spain, solving their power quality problems: datacenters, banking, healthcare facilities, newspapers, commercial buildings and different types of industrial plants.

In this paper, the power quality problems that have been found during these ten years will be summarised, providing sources of common errors, real data measurements, statistics and solutions.

\section{Field Experiences}

10 years have passed since PQC started its activity in the field of Power Quality. During this time, hundreds of electrical plants have been audited, from general audits to detect the inherent risks to direct resolution of problems that are affecting the plant, including forensic analysis of damaged plants.

Considering the concepts more often treated in these audits and which are in the origin of most of the detected problems, we can establish the following classification:

\begin{tabular}{|l|l|}
\hline $1^{\text {st }}$ Neutral Earthing System & $51 \%$ \\
\hline $2^{\text {nd }}$ UPS and Emergency Power & $18 \%$ \\
\hline
\end{tabular}

\begin{tabular}{|l|r|}
\hline Supplies & \\
\hline $3^{\text {rd }}$ Harmonic Distortion & $13 \%$ \\
\hline $4^{\text {th }}$ Transient Overvoltages & $7 \%$ \\
\hline $5^{\text {th }}$ Others & $11 \%$ \\
\hline
\end{tabular}

Table 1. Classification of the origin of Power Quality problems

\section{A. Neutral earthing systems}

It is the most common cause of power quality problems, as in more than $50 \%$ of the studied cases the neutral earthing system and the neutral distribution was the main cause of anomalies.

In the earthing of a plant, the following concepts must be considered:

\section{1) The Neutral System}

During a lot of years, the consecution of specific ohmic values in substations has been a goal to achieve, while the exact definition of the neutral system in the low voltage side has been widely ignored. In a vast amount of the projects examined by PQC, including the most recent ones, there is a lack of definition of these concepts, leading to plants being built based on the habits of the electricians. Even when in a rare case, a definition of these concepts was present in the project, the electrician, with no habit of dealing with definitions of this kind has finished its job following its habits instead of following what the project specified.

As a brief summary, the REBT [1] allows, both in 1973 and 2003 editions, the TT, TN and IT earthing systems.

- The TT is a system where the earthing of the neutral of the transformer is done isolated from the low voltage general earthing.

Apart from the public distribution in low voltage, where it is compulsory, and although regulations like the NEC forbid it, in Spain, for high voltage customers, it is the most widespread system $(61,4 \%$ of the plants analysed by PQC have this system).

The separation of both earths limits the maximum fault current, depending on the obtained ohmic value (in the studies performed 
by PQC, 62,9\%<4 ohms, 27,1\% between 4 and 12 ohms and only $10 \%$ above 12 ohms).

- In the TN system, the earthing of the loads is joined with the transformer neutral earthing, setting up a low impedance path for the fault currents. $35,1 \%$ of the plants which PQC has examined, are, supposedly, TN (a measurement which results in TN like values does not imply that this is the actual system).

- $\quad$ And last, the IT system (3,5\% of the cases) has the particularity of having the neutral isolated from earth, and so, a unique phase to earth fault does not act as a fault and the service is maintained.

\section{2) Substation Configuration}

In a Substation there is a protection earth and a service earth, besides a possible low voltage earth which relation with the neutral has been described earlier.

The relationship between the protection and service earths (joined or separate) is regulated by the RAT [2], while the relationship between high voltage and low voltage earths is regulated in the REBT, where, except for specifically addressed cases, total separation is recommended.

Most of the plants revised by PQC have high voltage and low voltage earths joined and a separate neutral earth. That is, most of the plants with a TT system have high and low voltage earths joined.

\section{3) Lightning arrester earths}

We have detected a great mess about this topic in the sector. Together with most recent regulations where it is said that the earthing of lightning arresters must be joined to the general low voltage earth, there is the habit of making a separate earth for the lightning arrester.

In many occasions we have been asked about which of the options is correct. The answer can be a surprise because it depends on the neutral earthing system. For a TN, the regulation is correct, but for a TT it is not appropriate.

\section{4) Independent earths in some equipment}

Although usually not a concern in new plants, PQC has analysed plants where there are a lot of independent earths. For example: high voltage, neutral, emergency standby generator, UPS, computers, phone, data...
Nowadays, an independent earth for equipment is a step backwards for the power quality requirements of the last generation systems. Some years ago, and in a wrong way, it was thought that an independent earth could provide a shielding to perturbations, but time has proved that it was an error.

The earthing system of a plant has a direct relationship with two fundamental topics of it:

- Correct operation of Equipment and Systems

It is the first goal of power quality. For the correct operation of electronic equipment, and in strict application of the fundamental power quality concepts, it is of uppermost importance that in the supply to them the "supply zero" and the "reference zero" be the same, that is, the lack of voltage between neutral and earth conductors.

To achieve this goal, the above topics must be perfectly combined, at least in the following way:

The neutral earthing system that most meets the necessities of current equipment, is the $\mathrm{TN}$, and more precisely, the TN-S, where the neutral conductor and the protection conductor are independent in all the circuits of the plant, from the common point placed in the earthing of the transformer.

The TT system, although it has some advantages considering its ease of application, is the most opposite system for the requirements of the equipment, because of the existence of important voltages between neutral and earth, and so, an open path for the electromagnetic noise towards the most sensitive equipment.

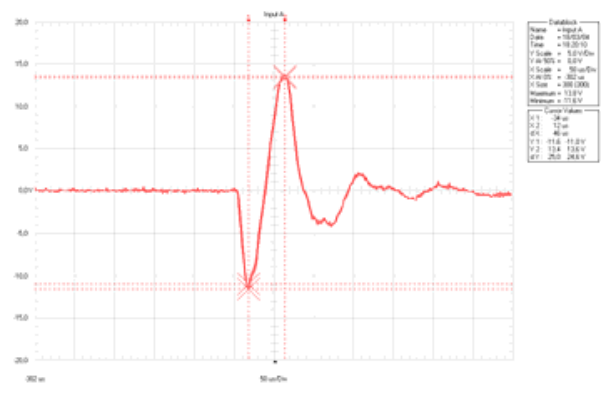

Figure 1. Electromagnetic noise in common mode.

And last, the IT system is not conceived for the equipment but for the continuity of supply. Current recommendations, and except very specific needs, also advise against the use of this system, from that point of view.

In the case of switching between grid and emergency standby generators, in Spain it is common to design them with 4 poles and neutral opening. The voltage transients that happen are a risk for the most sensitive equipment. 
The joining of high voltage and neutral earths and the connection of the lightning arrester earth to the same earth of low voltage, can affect the equipment in the case of a TT system. In a TN system, it will have no influence. But independent earths, are in any case, a problem.

In any case, and whatever the earthing system of the plant, it will always be possible to set up a local system to supply specific loads. By inserting a separate and parallel system, being its most common example the isolation transformer, a new neutral to earth reference is created.

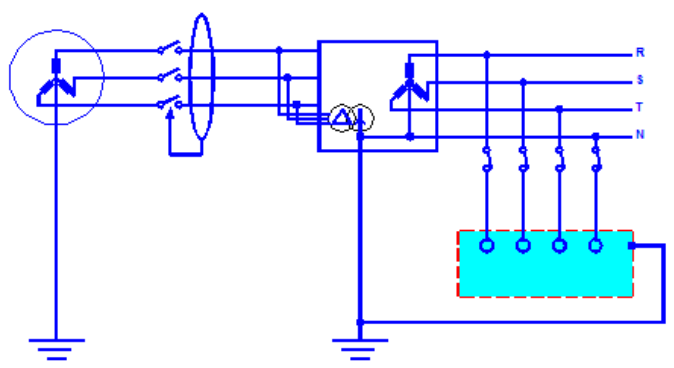

Figure 2. Isolation transformer.

- $\quad$ Safety of the people and the plant

Although not a topic associated to power quality, it would be useless to carefully select the supply system of a critical plant if the plant burns out or if people in contact with it are exposed to electrical shock danger.

From this point of view, we must inform that a very high percentage of the fires of electrical origin, in which PQC has had the opportunity to make a forensic analysis, have been caused by an unprotected phase to earth fault. It is, probably, the most dangerous risk to our electrical plants. Besides, the protection of indirect contact is generally, very badly solved, as in almost all of our studies there are points that involve a great danger for the people.

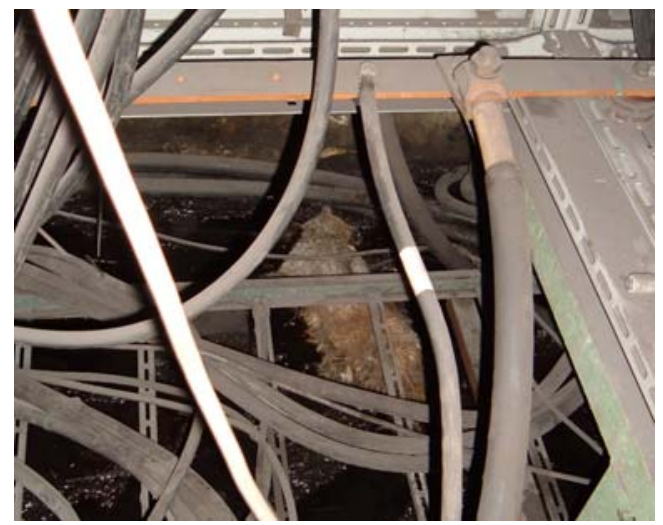

Photo 1. A cat corpse inside an electrical cabinet.

\section{B. UPS and Emergency Standby Generators}

The addition of UPS systems with Emergency Standby Generators represents for the user a security of supply for critical loads. However, the reality is very different.

The operation of these elements during their lifespan and their interaction creates risk scenarios for the plant. Below we summarise the most recent experiences of PQC in this field.

\section{1) Emergency Standby Generators}

The most common emergency standby generator is a combustion motor associated to a three-phase generator. From small powers to values up to 2000 kVA, we have had the opportunity to analyse different features of their operation, besides studying the origin of their malfunction in times when they were needed the most.

The causes of their malfunctions are, among others and classified from high to low probability:

- $\quad$ Failure to start up (batteries)

When there is no supply voltage, the generator must start up drawing power from the batteries, which are not always in optimum conditions. A bad maintenance is a frequent cause of this type of anomaly.

- $\quad$ Alarm stop (fuel or smoke)

Due to a lack of control of present levels and a lack of actual tests in which the system thermal balance is reached.

- Incompatibility with feeders

It is very common to find generators which are feeding non linear loads, with a rich harmonic current content. This condition, if it is not taken care of with a de-rating of the power to be delivered, leads to a distortion of the voltage waveform of the generator and can be incompatible with equipment needs.

Besides, we must quote the risk of placing capacitor banks in critical load circuits. This happens when there is no separation between busbars and the disconnection of the capacitor banks is done by means with high failure rate.

\section{- Overload}

In plants where loads have increased with no control, and where the original design of the standby generator was small, a state can be reached where the generator is not able to cope with the loads and stops. 


\section{2) Switching}

The grid-generator switching is in a tight relationship with the emergency standby generator. Although the generator has started up correctly, there are lots of causes in which, a switching failure prevents the voltage switching to the loads. Among them:

\section{- Electromechanical failure}

It is the most common cause as these systems have a moving part which is prone to failures in many of its components.

- Switch in manual position

We have analysed many cases in which maintenance staff was not able to correctly operate a system that was, unintentionally, in a non automatic position.

- $\quad$ No reclosure after a UPS failure

This last cause is lately becoming more common. By feeding the switching circuits from the "secure" voltage of the UPS, the emergency standby generator is unable to start up when there is a failure in the UPS system (and sometimes even the grid when grid supply returns).

\section{- Nuisance tripping of protections}

When there is an opening of the neutral during the grid to generator transfer, or generator to grid transfer, that is, when the switching system is of four poles, there are many combinations of neutral earthing systems of each part, which in most cases leads to the apparition of transient neutral to earth voltages of important level. These levels usually are in the origin of nuisance tripping of protections.

\section{3) UPS}

Except few exceptions (flywheels, motor-generator groups, etc.), most of the loads that, nowadays, are considered of critical importance, are being supplied by static battery UPS systems.

For many customers, their use has been synonym of security, but experience has proved that these systems have weak points:

\section{- $\quad$ Batteries}

Although batteries are a well known technology, battery operation is a world scale problem, as has been stated by different countries in Power Quality Conferences of last four years which PQC has attended.

In Spain we are no exception and the cases in which a failure of supply to a critical system has been due to a malfunction in the batteries, are very numerous and some of them very scandalous.

The incorrect placing and maintenance of batteries (temperature, humidity...) and the lack of testing and monitoring are some of the causes that lead to failure. The wearing out of some of the batteries or the breakdown of a single cell are the first effects.

As an example, figure 3 below shows the influence of temperature in the life of a battery and figure 4 shows what happens to the battery voltage when the battery is suddenly required to supply its rated power. The initial voltage drop can cause the UPS system to disconnect because the voltage level falls below the minimum level required for proper operation of the UPS.

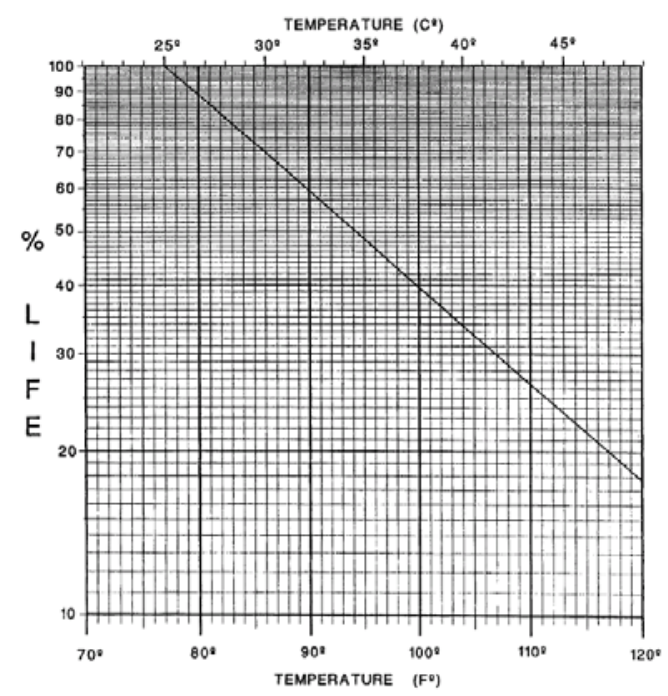

Figure 3. Influence of temperature in the life of a battery. Extracted from [3]

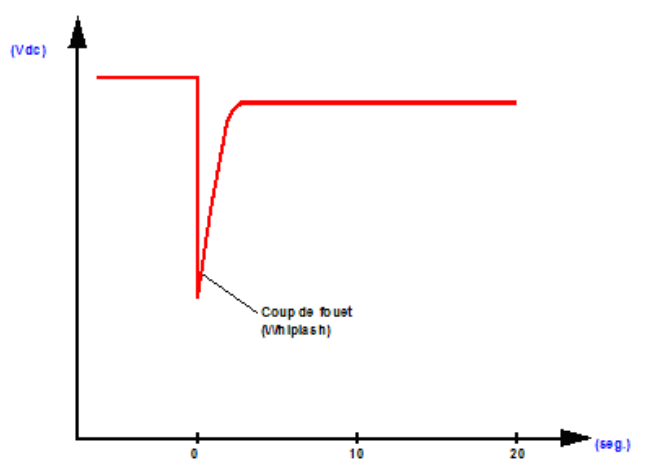

Figure 4. The whiplash that happens when a battery is required to supply a rated power suddenly.

- The bypass circuit and protection coordination

Both in single and multiple parallel UPS systems with single or multiple bypass circuits, the operation of the general protection during a short circuit in a partial circuit has led to system halts.

Coordination problems between protections (both by their settings and by the selection of their features), 
are affecting, not only to generic plants, but also to those which depend on an uninterrupted supply.

\section{- $\quad$ Single Points of Failure. Redundancies}

According to the Uptime Institute, the vast majority of the failures that affect to the supply of the most critical equipments (their study has been made with data from 1700 datacenters) happen between the UPS and the supply to critical equipments, that is, in the distribution circuits. As a result, all the effort has been directed to the use of redundant systems in order to eliminate the single points of failure.

However, during last years, the redundancy concept has been applied, in our opinion, in a wrong way, granting more importance to the UPS itself at the sacrifice of the part of the circuit where failures happen most frequently. And because of this, the singular points of failure do not disappear from the plants.

\section{- “Other” features}

Not in great plants, but in smaller ones, the concepts being considered when adding UPS systems have been the power and autonomy of the system. However, there are other equally important concepts, which if not considered from the beginning, will end up being essential. Among those concepts, we consider:

-Transient overload capacity

-THD of input current

-THD of output voltage

-Type: off-line, double-conversion or interactive

-Efficiency for different load levels

-Common mode shielding

\section{Harmonic distortion}

Harmonic distortion is lately one of the most quoted concepts whenever electrical problems arise in a plant.

Although a harmonic distortion study can be sometimes very difficult to perform (technical references are full of complex equations of difficult understanding for the plant staff), the first approach must be done in an easy way.

\section{1) Fist step}

We always start with an analysis of the harmonic sources and the study of its first consequences which usually affect to transformers and conductors (overheating), and to generators (instability).

The most common one is the case of transformers, where the flow of a non linear current produces a much greater heating than the one which would produce a linear current of the same rms value. This leads to the need of de-rating the transformer, limiting its use to a power level lower that the rated one.
If this is considered in the design phase, a K-rated transformer can be ordered. The K-Factor in USA or Factor-K in Europe, stands for a transformer specifically manufactured to withstand non-linear currents while de-rating a transformer implies using a "common" transformer below its rated power to compensate the additional losses caused by harmonic currents.

\section{2) Second step}

Once the power requirements for the harmonic loads of the plant have been verified, the second step consists in the checking of voltage harmonic levels. Harmonic currents, when flowing through elements with an impedance, produce voltage drops which, if dependant on the frequency, cause a distortion in the voltage waveform.

And so, for example, even if a transformer has enough thermal margin to withstand a certain amount of harmonics, its internal impedance affects the secondary voltage. If this disturbance is important, all the loads supplied from that point will be affected.

Although the EN50160 limits the maximum allowable voltage harmonic distortion in $8 \%$, our recommendations about this topic are: if the voltage distortion level is below 3\%, we can consider that there are no risks; if the level goes above $5 \%$, it is advisable to carefully control the plant; and if the level nears or exceeds $10 \%$, then the situation is dangerous and immediate measures should be taken.

\section{3) Third step}

The third step consists in checking, beginning with the voltage distortion level, the waveform of linear loads which waveform will be identical to the voltage waveform. At this point it is necessary to take into account the harmonic sequence (positive, negative or zero), because for example, the $5^{\text {th }}$ harmonic, quite common, is of negative sequence and so, in a motor (linear load) will cause a torque opposite to the motor torque, increasing the starting current and so causing a overheating.

In PQC we have received many queries about harmonic distortion in plants, and have started many field works with the assignment of the client to "solve the harmonics problems”. However, although we cannot deny that harmonics are important, in a high percentage of studied cases, the anomalies of the plant had nothing to do with harmonics. And so, based in our experience, we can establish the following starting recommendations:

-Do not thing necessarily that any new unaccountable problem is due to harmonics.

-Do not consider harmonics a scapegoat.

-Do not mix up voltage and current harmonics 
-Do not suppose that a high harmonic content leads necessarily to risk situations

-But if despite all, the problem subsists, take the needed measures to solve it out.

As it has been said before, the presence of harmonics does not always imply a problem and not always the existing problems are caused by harmonics. However, we have dealt with many cases where actually harmonics were negatively influencing the plant. In most of those cases, it was a resonance problem.

A resonance is produced when, for a given frequency, some of the circuits adopt a minimum impedance value, producing a high increase in the flow of currents of that given harmonic through those circuits. The most common case occurs with power factor correction capacitors.

The resonance frequency is directly proportional to the power of the supply transformer, and inversely proportional to the capacitive power and to the short circuit voltage of the transformer. If the resulting value matches some of the values of the loads, the resonance will have two effects:

- A high increase of the currents of that given frequency through the capacitors and the transformer.

- An increase in the voltage harmonic distortion of that frequency, which, sometimes, can reach very high levels (we have sometimes measured values well above $20 \%$ ).

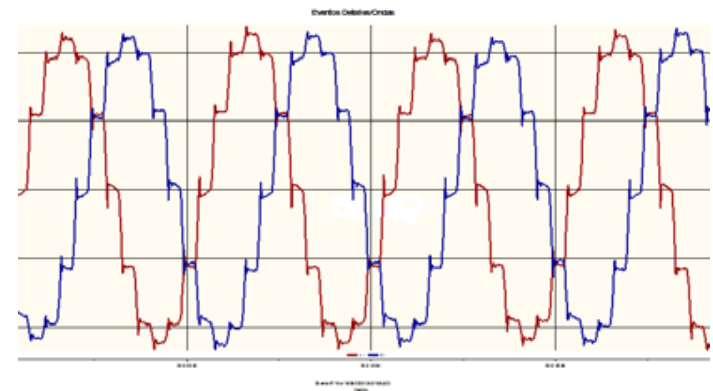

Figure 5. Harmonic distortion measured in a plant.

\section{Transient overvoltages}

As last generation electronic loads are being placed in all types of plants, their advantages are clearly perceived. The most obvious ones are:

-Higher processing speed

-Higher memory capability

-Better withstand of voltage sags

-Higher voltage tolerance levels

-Higher frequency tolerance levels

However, together with these advantages that make it impossible ignore the purchase of these equipments, there are some drawback, being the most important one their operation in the presence of transient overvoltages.

Overvoltages, both the ones caused by lightning strikes and the ones caused by disturbances in the supply line or faults in the plant, have been always present. However, it is the introduction of sensitive electronic equipment which has made the study and eradication of these disturbances an essential goal.

Wherever the overvoltage is generated, the objective is to avoid it reaching sensitive equipment, and this can be accomplished by rejection (filters in series) or by derivation (elements in parallel).

In Spain, and except some small signalling circuits, the serial protection is not used, being the most common one (although in very few plants) the parallel protection by the use of surge suppressors.

In this type of protection devices, the incident overvoltage is derived by the placement of an element whose impedance is variable with voltage. From a given threshold, the element starts conducting and a discharge current is created, leaving only a residual voltage to sensitive equipment.

The experience in the analysis of transient overvoltage protection devices has given us some important facts to be concerned about:

- There are still very few plants with overvoltage protection devices.

- In the few plants where they are present, their installation is incorrect.

- Sometimes, their presence is an added menace instead of a solution.

- $\quad$ The fact that the new REBT has an specific technical instruction about this topic has lead to the publishing of a Technical Guide which includes states that the use of these protections are obligatory for a lot of different types of plants. If the installation of these devices is done as it has been done till now, we do not foresee a good future.

\section{E. Monitoring}

Nowadays, the capabilities of power quality monitoring equipment have reach such a high level, that they provide quite a lot more information than the one that is required. As the price of very advanced devices has dropped a lot, it is becoming quite common to find these type of devices in many plants, or electricians which have one device, because of the new REBT, but who do not know how to use it.

We have found customers who bought top class power quality monitoring equipment and have it stored because they are unable to use or understand it. 
But, which is in our opinion worse, the lack of qualified professionals to interpret the data that this equipment provides, has lead some companies to make very extensive reports where they only put measured data but do not make an analysis of that data, and so they provide useless reports with no value for the customer.

\section{Conclusion}

We have presented a summary of our field experience in dealing with power quality problems of final customers. We think that an effort should be made in spreading some basic concepts to plant engineers because as we have stated in the preceding pages, most of the causes of power quality problems in final customers are in the origin, well known concepts in the academic world but not for plant engineers and maintenance staff.

We have detected the same problems in other countries, and in references [4], [5] and [6], it can be seen that the same concepts are being explained in other countries.

\section{References}

[1] REAL DECRETO 842/2002, de 2 de agosto, por el que se aprueba el Reglamento electrotécnico para baja tensión. Reglamento Electrotécnico para Baja Tensión.

[2] DECRETO 3151/1968, de 28 de noviembre, por el que se aprueba el Reglamento de Líneas Eléctricas Aéreas de Alta Tensión.

[3] IEEE Std 1184-1994, "IEEE Guide for the Selection and Sizing of Batteries for Uninterruptible Power Systems”

[4] David Mueller, "Troubleshooting and Solving Power Quality Problems”, Power Quality Exhibition \& Conference, 2005, Baltimore, MD.

[5] David Brender, "Wiring and Grounding for Power Quality”, Power Quality Exhibition \& Conference, 2005, Baltimore, MD.

[6] Stefan Fassbinder, "Power Quality and Non-linear Loads”, Leonardo Power Quality Initiative, www.lpqi.org 